\title{
Linguistic Development in Stuttering Children
}

\author{
M. Pamplona ${ }^{*}$ A. Ysunza and F. González
}

Hospital General Dr. Manuel Gea González, Mexico

\begin{abstract}
Normal fluency errors usually appear during language development, especially during the transition between the stages of one-word and simple phrases. In cases of stuttering the speaker produces several involuntary repetitions of phonemes, syllables, or words. The speaker can also show phoneme prolongation or a complete interruption of speech production. Other abnormal fluency failures in stuttering include tension pauses and fragmented words. An individual with stuttering can show associated related behaviors and movements, including tremor, eye blinks, and hand movements, among others.
\end{abstract}

Aim: To study the relationship between language development and organization and the presence of stuttering in children.

Materials and Methods: Twenty children with stuttering, age ranging from 4 to 7 years old were studied. A matched control group of 20 children without stuttering and without a history of language impairment were also studied. For evaluating language development, all patients were analyzed using the Situational- Discourse- Semantic (SDS) Model. This model is a valuable tool for conducting naturalistic observation and descriptive assessment of language development. The SDS Model provides a detailed description of 3 language contexts (situational, discourse and semantic) in 10 levels of cognitive and linguistic organization.

Results: In all contexts considered by the model of cognitive and linguistic organization used for this study, i.e. situational-discourse-semantic, a significantly higher frequency of language delay was demonstrated in stuttering patients, as compared with patients without stuttering. Furthermore, none of the patients present with stuttering showed an adequate level of language development.

Conclusion: Children present with stuttering demonstrated a significantly higher frequency of delay in language development as compared with children without stuttering. From the results of this paper, it seems that a detailed evaluation of all aspects of cognitive and linguistic organization should be performed in children with stuttering. Moreover, it seems that speech intervention in stuttering children should address not only fluency, but also specific aspects of language development.

\section{INTRODUCTION}

Fluency is the natural ease, rate, and continuity of speech production.

Fluent speech is continuous, natural, and its speed is quick and efficient. In the fluent discourse, speech is efficiently produced. Appropriate speech includes an adequate vocabulary and social accepted emissions. The speaker selects the correct word order in order to form sentences. The fluent speaker stops for thinking the words he will use, repeat some word, or show a failed start only occasionally. Failure in speech fluency includes the involuntary repetition of the initial syllable or phoneme of a word [1]. At times, an individual can hesitate when the word or order of words are selected. These hesitations have also been described as a failure in language fluency [2].

During speech and language development, there is frequently a stage in which the child produces regular, effortless, repetition of initial syllables, words, or phrases. The child can also show silent pauses, and use interjections or revisions. During this stage, the child does not seem aware that his/hers fluency is not perceived as natural and normal.

*Address correspondence to this author at the Department of Phoniatrics, Hospital General Dr. Manuel Gea González, Mexico, D.F.;

E-mail: santypa@prodigy.net.mx
Approximately 1-3 \% of children with language development within normal limits show these types of failures [3].

In contrast, stuttering is a disorder of rhythm \& timing in which the speaker knows what he/she wishes to say, but for a brief period of time he/she cannot express his/hers thoughts. In these cases, the speaker produces several involuntary repetitions of phonemes, syllables, or words. The speaker can also show phoneme prolongation or a complete interruption of speech production. Other abnormal fluency failures in stuttering include tension pauses and fragmented words. An individual with stuttering can show associated related behaviors and movements, including tremor, eye blinks, and hand movements, among others.

Stuttering children characteristically show more than $10 \%$ of abnormal speech fluency failures during a conventional conversation [4].

There has been significant controversy regarding stuttering management in the related scientific literature. Several treatment modalities have been proposed for modifying and correcting fluency in stuttering persons. It has been suggested that early intervention seems more efficient [5].

During the last 20 years, there have been several reports concerning various techniques for modifying speech rate and rhythm for treating stuttering children. These modalities have been designed for restoring a normal sound and rhythm 
of speech. These programs are based upon the modification of emission speed until a normal range can be achieved [6].

Brady, in 1971, studied several treatment programs based upon rhythmic stimulation. These programs use rhythm of speech and also rhythm in areas not necessarily depending on speech, including psichomotricity and discrimination of musical sounds. Several drills with voiced reading following a rhythm are used [7].

Other programs have been based on biofeedback and emission analysis. In these programs, the patients analyze their own speech production and compare the natural speed and pauses during speech [8].

These and other techniques are aimed to modify the speech fluency of stuttering patients. These treatment modalities are focused on language form, that is, emission of words, relationship between words, and production of speech sounds. However, in these procedures, the inner processes which occur during oral communication are not addressed. In order to elaborate and comprehend a message or discourse, several cognitive and linguistic variables have to be taken into account. These variables determine coherence and order in the inner areas of thought. These inner processes are known as linguistic organization [9].

Several children with less flexible language systems show failures in their inner linguistic structure. As a result, these children show a wide range of problems in pragmatic language. These children can understand what is going on within an event but they failed to organize and interpret the experience. Hence, it is difficult for them to learn of the experience or make generalizations for new events.

A person taking part in a conversation must coordinate three interacting knowledge contexts - the situational, discourse, and semantic. The person engaged in a conversation accesses his cognitive knowledge of the topics being discussed. The first topics that a child is able to talk about are contextualized to the people, objects and actions in the social or physical situation that he is currently in. With development, the child is able to think and talk about decontextualized situations that occurred in the past or might occur in the future. The child must also develop the ability to talk about increasingly more abstract symbols including pictures and print. The person organizes his utterances to fit into narrative and expository discourse structures. These structures allow the person to keep track of what has already been said about a particular topic within a conversation. Children develop the ability to organize topics to include time then causality, then intentional planning. Each utterance must be organized at an appropriate level of semantic complexity to fit the current point in the discourse. Simpler utterances interpret and infer emotions, plans, and predicted past or future events. As semantic complexity increases, utterances include more complex morphology and syntax. Children who stutter produce more disfluencies when talking about decontextualized topics than contextualized topics [10].

Speech therapy focusing on linguistic form offers some intrinsic advantages. Appropriate forms for each age can be easily selected in order to be used for teaching objectives according to the child development. Nonetheless, some speech pathologists have realized that language goes beyond form, including several elements such as morphology, phonology, pragmatic, and syntax [11].

Thus, speech should express use and content as well as form. Speech and language intervention should be based on meaning with a social orientation. Intervention should address the various areas of language within meaningful contexts where language is considered a whole for communicative purposes [12].

Considering these elements, it seems reasonable to think that there can be a relationship between speech fluency and linguistic organization.

The purpose of this paper is to study whether stuttering children show a significantly higher frequency of linguistic organization failures.

\section{MATERIALS AND METHODS}

Children in whom the diagnosis of stuttering had been established were compared with a matched group of subjects without stuttering and without a history of language impairment. The comparison was made for language abilities in the contexts of free play and story retelling. Language samples were analyzed for the semantic level of ideas expressed, discourse organization, and the level of situational displacement, structure, and organization.

\section{Subjects}

The experimental subjects for this study were 20 children in whom the diagnosis of stuttering had been made. Subjects were between the ages of 4 and 7 years at the time of evaluation (mean=5.2 years, $\mathrm{SD}=1.44$ ). A matched control group of 20 children without stuttering and without a history of language impairment was also identified. Both groups of subjects were recruited in the phoniatrics department of the Hospital General Dr. Manuel Gea González at Mexico City from January 2001 to December 2003. To qualify for the experimental group for this paper the patients had to meet the following criteria:

1) Stuttering. The diagnosis of stuttering was performed using the Identification of fluency failures in children test. A score of $>10 \%$ failures during conversation in a free sample of 100 words was considered as indicative of stuttering [4].

2) No known neurological or genetic syndromes

3) Chronological age between 4 and 7 years at the time they were selected for the study.

4) Normal hearing demonstrated by conventional puretone audiometry.

Twenty children who met these criteria were included in the experimental group.

A control group including children of similar age, without stuttering as demonstrated by a score within normal limits in the test of Identification of fluency failures in children, and without a history of language impairment was matched for the dimensions such as gender, educational level of parents, and socio-economic status. They were selected from the Day Care Center of the Hospital Gea González. In this center, children of the employees of the Hospital are admitted 
for providing day care. The ages in the control group ranged from 4 to 7 years $($ mean $=5.8, \mathrm{SD}=1.91)$.

To determine if the groups were equivalent and homogeneous, a student t-test was run for chronological age. Also, U-Mann Whitney Tests were run for the ordinal variables (social/economical status and educational level of the parents).

\section{Evaluation}

For the diagnosis of stuttering the Identification of fluency failures in children, was applied to all subjects participating in this study [4].

To determine if there were differences in the language abilities between children with stuttering and controls, language samples were elicited from both groups. The samples were obtained under the naturalistic condition of storytelling. All interactions were video recorded for later transcription and analysis.

\section{Sampling}

Each child met individually with one of three trained examiners for approximately 30 minutes of story time. Stories were elicited from one of two sources that had two different levels of complexity. All subjects were shown a storybook about a familiar topic. It was about a boy that got lost in a store. The story had a narrative structure and the pictures had a lot of details that help follow the ideas and actions. Children saw the story with the examiner and were asked to look at the pictures and participate in telling the story. They were given question prompts to help elicit more complex information or details. If necessary, the examiner modeled the reconstruction of the story. This model included more complex ideas and better story organization than those produced spontaneously by the children. Subjects were then asked to retell the modeled story from the pictures. Both the spontaneous story and the retelling were videotaped and analyzed.

In the case of the youngest subjects, this story was too difficult and failed to elicit sufficient language production for analysis. In these cases, a second storytelling was elicited using an illustrated storybook about bath time which told a boy getting ready for bed and taking a bath, but making a mess at every step of the process. Once again the procedure of eliciting a spontaneous telling, question prompts, and modeling followed by retelling was used.

\section{Transcription}

All videotaped interactions were transcribed verbatim, including the conversational turns of the examiner and the child. Three examiners transcribed the samples which were randomly assigned to an examiner. Each transcription was then checked against the videotape by a researcher that had not done the original transcription along with a second examiner for accuracy. If either of these two judges differed from the transcription, the videotape was watched again until consensus was reached. All videotapes for all subjects were verified for accuracy in this manner.

\section{Coding}

For obtaining a specific level of linguistic organization, each sentence was coded for the level of meaning (seman- tics) expressed using the criteria established in the Situational-Discourse-Semantic model [9]. In this model, the level of representation present in the activity is specified in the Situational context. If the child could interpret and talk about the pictures, this was scored as Level $4=$ symbolic representation. If the child only played with the book but did not understand the picture symbols, a score of Level $3=$ relational actions was assigned. This same criterion was applied to the play house activity. Level 4 represented symbolic play, meaning the child was able to make the characters perform actions and talk. Level 3 represented non symbolic actions such as holding the characters, stacking furniture pieces, or doing only single-actions such as rolling a car.

The Semantic context was scored for the level of meaning expressed during story telling or play. The samples were evaluated for the highest level of meaning expressed by the child. In the SDS model, the first 2 levels are nonverbal responses, and these were not scored. The lowest scored was Level 3, Labeling, which was assigned to a communicative turn when the child only named a toy or something in the picture (e.g., "There is a car." "I see the dog.") Level 4, Description, was assigned if the child talked about actions, as in "Go to sleep now," or "The boy is taking a bath." Level 4, Attribution was assigned if the child described characteristics or emotions, such as "My car is rolling fast" or "The mother is sad." Level 5, Interpretation, was scored if the child made a prediction or mentioned causality or similar insight, as in "I think it is going to crash" or "He is going to sleep because he is very tired". The highest score assigned, Level 6 was given if the child made an inference that required combining personal experience or prior knowledge with the action, as in "He is going to be in trouble because he is hiding instead of going upstairs to eat dinner." The child was assigned the Semantic level equivalent to the highest produced that occurred with high frequency (i.e., more than five occurrences) during play and retelling.

The Discourse was scored according to the highest level of organization shown in the play and story telling. Level 1, a single discrete action with no continued interest was not seen by any of the subjects. Level 2 was assigned to a loose collection of actions or comments with no overall theme or topic shown. Level 3 was assigned to organization in list form, as in "There is a mommy, and there is a dog, and he is taking a bath, and she is hiding" with no temporal connections. Level 4 was assigned if temporal connections were made between events, as in "He is taking a bath and now he will dry off. Then the boy brushed his teeth." Level 5 was assigned when causality was established between actions, as in "Dad cooked dinner while mom did the laundry. Dad called everyone to dinner, but the boy did not come because he was hiding. Dad became very angry." Levels 4 and 5 of Discourse required temporal or causal links across actions or events, and therefore required the child to play or tell a sequence of at least 3 related actions. The child was assigned the Discourse level equivalent to the highest produced during play and retelling.

The coding resulted in one number assigned for Situation, one for Discourse, and one for Semantics for each subject. The first coding was done by one of three randomly assigned examiners. A second researcher also coded the samples for all three measures. Intercoder agreement was .95 . 


\section{Scoring}

The samples were analyzed by comparing the child's assigned levels to those expected at age level. The SDS Model provides age norms for each level of development based on typically developing children.

The child's profile for the Situational, Discourse, and Semantic aspects of language were scored by subtracting the assigned level obtained from the assessment from the expected level established by the SDS age norms. This resulted in a number score, ranging from 0 to 4 , that represented the number of levels of discrepancy or delay. For analysis, a score reflecting a delay (i.e., a discrepancy of one to four levels) was classified as "delayed." A score of zero levels of discrepancy represented typical performance, meaning the child performed at an age appropriate level. The Situational, Discourse, and Semantic aspects of language then were compared statistically for differences between the experimental and control groups.

\section{RESULTS}

A student- $t$ test demonstrated that there was a nonsignificant difference $(p=0.263)$ between the ages of the patients from the active, and the control groups. Moreover, a Mann-Whitney test demonstrated non-significant differences between both groups of patients for social-economical status and educational level of the parents.

For the stuttering test, all children from the experimental group showed $>10 \%$ of abnormal speech fluency failures such as tension pauses, repetition of phonemes and syllables, and fragmented words. In contrast, none of the children from the control group showed pathological disfluencies. Only some of them showed normal fluency failures such as word repetitions or false starts. The scores of the three contexts of language were statistically compared between the experimental and control groups. The discrepancy scores between expected level of performance and actual level represented ordinal data.

Table 1 profiles the number and percentage of subjects in the experimental and control groups according to the number of levels of delay obtained for the Situational context. This table shows that while no children in the experimental condition showed typical development (i.e., a discrepancy score of $0), 15$ of the control children (75\%) scored at their age level. The minority of the experimental children showed small delays, with $40 \%$ scoring either one or two levels below age norms. A larger number of children (twelve) were far below expected levels with scores of three and four levels below age norms $(60 \%)$. In contrast, none of the control children scored more than one level below age norms, indicating that all delays shown were small. To determine if these represented significant group differences, a U-Mann-Whitney Test was conducted. An alpha value of $p<.05$ was selected for considering the results as significant. Results indicated that there was a significant difference between the groups $(\mathrm{p}<0.001)$.

Table 2 profiles the number and percentage of subjects in the experimental and control groups according to the number of levels of delay obtained for the Discourse context. This table shows that while no children in the experimental condi-
Table 1. Situational-Discourse-Semantic Model of Linguistic Development [9] Levels of delay Situational Context

\begin{tabular}{|c|c|c|}
\hline Patient No. & Group I (Experimental) & Group II (Control) \\
\hline 1 & 2 & 0 \\
\hline 2 & 1 & 0 \\
\hline 3 & 1 & 0 \\
\hline 4 & 1 & 0 \\
\hline 5 & 2 & 1 \\
\hline 6 & 3 & 0 \\
\hline 7 & 4 & 1 \\
\hline 8 & 4 & 0 \\
\hline 9 & 4 & 0 \\
\hline 10 & 3 & 0 \\
\hline 11 & 4 & 0 \\
\hline 12 & 4 & 1 \\
\hline 13 & 3 & 1 \\
\hline 14 & 1 & 0 \\
\hline 15 & 4 & 0 \\
\hline 16 & 2 & 0 \\
\hline 17 & 4 & 0 \\
\hline 18 & 1 & 0 \\
\hline 19 & 4 & 1 \\
\hline 20 & 3 & 0 \\
\hline \multicolumn{3}{|c|}{$\begin{array}{l}25 \% \text { showed } 1 \text { level of delay } \\
15 \% \text { showed } 2 \text { levels of delay } \\
20 \% \text { showed } 3 \text { levels of delay. } \\
40 \% \text { showed } 4 \text { levels of delay. } \\
T=222.5 \\
Z \text { sub } T=5.255 \\
p=0.000 \\
p<0.001\end{array}$} \\
\hline
\end{tabular}

tion showed typical development (i.e., a discrepancy score of $0), 17$ of the control children $(85 \%)$ scored at their age level. Eight children of the experimental group showed small delays, with $40 \%$ scoring either one or two levels below age norms. Twelve children (i.e., 60\%) were far below expected levels with scores of three and four levels below age norms. In contrast, only one of the control children scored more than one level below age norms. A U-Mann-Whitney demonstrated that both groups showed a significant difference in this context $(\mathrm{p}<0.001)$.

Table 3 profiles the number and percentage of subjects in the experimental and control groups according to the number of levels of delay obtained for the Semantic context. This table shows that as in the two previous contexts no children in the experimental condition showed typical development (i.e., a discrepancy score of 0 ). Fourteen of the control children $(70 \%)$ scored at their age level. For this measure only $30 \%$ of the experimental children showed small delays. Seventy percent of the subjects performed three or four levels below age norms. In contrast, $30 \%$ of the control subjects 
Table 2. Situational-Discourse-Semantic Model of linguistic Development [9] Levels of Delay Discourse Context

\begin{tabular}{|c|c|c|}
\hline Patient No. & Group I (Experimental) & Group II (Control) \\
\hline 1 & 3 & 0 \\
\hline 2 & 1 & 0 \\
\hline 3 & 2 & 0 \\
\hline 4 & 2 & 0 \\
\hline 5 & 3 & 2 \\
\hline 6 & 4 & 0 \\
\hline 7 & 3 & 1 \\
\hline 8 & 4 & 0 \\
\hline 9 & 4 & 0 \\
\hline 10 & 2 & 0 \\
\hline 11 & 4 & 0 \\
\hline 12 & 3 & 1 \\
\hline 13 & 3 & 0 \\
\hline 14 & 2 & 0 \\
\hline 15 & 3 & 0 \\
\hline 16 & 2 & 0 \\
\hline 17 & 4 & 0 \\
\hline 18 & 2 & 0 \\
\hline 19 & 4 & 0 \\
\hline 20 & 2 & 0 \\
\hline \multicolumn{3}{|c|}{$\begin{array}{l}10 \% \text { showed } 1 \text { level of delay. } \\
5 \% \text { showed } 2 \text { levels of delay. } \\
85 \% \text { within normal limits. }\end{array}$} \\
\hline
\end{tabular}

performed below age norms, all showing small delays (i.e., one or two levels). To determine if there was a significant difference, a U-Mann-Whitney Test was conducted. Results indicated that the scores of both groups were significantly different for this measure $(\mathrm{p}<0.001)$.

\section{DISCUSSION}

The purpose of this paper was to explore the relationship between stuttering and the child's language abilities. It was proposed that stuttering children would differ in their overall development of language, and not just in their fluency of speech, from children who do not show stuttering. The results of this study seem to support this statement. Children with stuttering were significantly different from those without fluency disorders in all three measures of language. Furthermore, all stuttering patients demonstrated at least one level of delay in language measures, while the majority of the subjects without stuttering performed accordingly with age norms. Only subjects in the experimental group scored more than two levels below age norms, with high percentages of stuttering patients showing these levels of delay.
Table 3. Situational-Discourse-Semantic Model of linguistic Development [9] Levels of delay Semantic Context

\begin{tabular}{|c|c|c|}
\hline Patient No. & Group I (Experimental) & Group II (Control) \\
\hline 1 & 2 & 0 \\
\hline 2 & 1 & 0 \\
\hline 3 & 1 & 0 \\
\hline 4 & 2 & 0 \\
\hline 5 & 3 & 1 \\
\hline 6 & 3 & 0 \\
\hline 7 & 3 & 2 \\
\hline 8 & 4 & 0 \\
\hline 9 & 4 & 1 \\
\hline 10 & 3 & 0 \\
\hline 11 & 4 & 0 \\
\hline 12 & 3 & 1 \\
\hline 13 & 3 & 1 \\
\hline 14 & 3 & 0 \\
\hline 15 & 3 & 0 \\
\hline 16 & 2 & 0 \\
\hline 17 & 4 & 0 \\
\hline 18 & 2 & 0 \\
\hline 19 & 3 & 2 \\
\hline 20 & 3 & 0 \\
\hline \multicolumn{3}{|c|}{$\begin{array}{l}20 \% \text { showed } 1 \text { level of delay. } \\
10 \% \text { showed } 2 \text { levels of delay. } \\
70 \% \text { within normal limits. }\end{array}$} \\
\hline
\end{tabular}

Many authors agree that stuttering is a multidimensional disorder [13]. For producing language efficiently, there are many factors that interfere directly and/or indirectly. However, few approaches take on account the different areas of development as a whole. Most approaches for intervention considers stuttering as a rhythm or word disorder and intervention focus on the form of language, respiration, or rhythm for promoting changes in fluency. These approaches do not consider inner processes for language organization and production. In contrast, we believe that linguistic organization can be an important factor that can affect fluency and speech.

It has been described that children's speech production is related not only to motor skills, but also to higher organizational levels of language processing [11]. The results from this study seem to support this statement. All patients with stuttering showed linguistic organization below the expected level according to chronological age in all three contexts considered by the model used for assessing linguistic performance in this study, i.e. situational-discourse-semantic. Moreover, it was observed that stuttering frequently increased when children were speaking in a decontextualized situation, for example, when they where retelling a story. In 
these situations they did not have the support of the pictures and they had to create the whole situation only with language. This makes the linguistic target a more abstract and complex task [10].

From the results of this study, it seems that a detailed evaluation of all aspects of cognitive and linguistic organization should be performed in children with stuttering. We consider that speech intervention in children present with stuttering should address not only fluency, but also specific aspects of language development. Hill [14] supports this idea when he stated that fluency disorder can not be treated in isolation because the over-arching goal of treatment is to facilitate development of fluency skills that are integrated with the ongoing processes of speech and language, social, and motor development. Other authors also considered that stuttering may be related with some linguistic aspects $[1$, $15]$.

The study group of this paper showed significant linguistic organization deficiencies. They all showed levels of delay in all measures of language. Also, we registered fails to predict, infer or evaluate situations, fails to make appropriate temporal, spatial, and causal relationships, difficulty to retell in a complex and organized manner the information revised previously, and difficulty to state possible consequences or changes in state, among others. These characteristics support the idea that stuttering children in this study seem to show difficulties to process and produce abstract and complex language.

The whole language model states that all components of language (phonology, morphology, syntax, semantics, and pragmatics) are simultaneously present and interacting in any instance of language use [16]. Thus, speech intervention in stuttering children should approach language as well as fluency. The whole language model seems a reliable tool for improving language organization deficiencies in stuttering children.

\section{REFERENCES}

[1] Starkweather CW. Issues in the efficacy of treatment for fluency disorders. J Fluency Disord 1993; 18:151- 168.

[2] Van R. The nature of stuttering. $2^{\text {nd }}$ ed. New Jersey: Englewoods Cliffs; 1985.

[3] Meyers SC, Freeman FJ. Interruptions as a variable in stuttering and disfluency. J Speech Hear Res 1985; 28: 428-435.

[4] Meyers SC. Nonfluencies of preschool stutters and conversional partners: Observing reciprocal relationships. J Speech Hear Disord 1989; 54: 106-112.

[5] Conture EG. Stuttering: It's nature, diagnosis, and treatment. Boston: Allyn Bacon; 2001.

[6] Curlee RLF, Perkins WH. Conversational rate control therapy for stuttering. J Speech Hear Disord 1969; 34: 245-250.

[7] Brady JP. Metronome-Conditioned speech retraining for stuttering. Behav Ther 1971; 2: 129-150.

[8] Inghaman I. Stuttering treatment efficacy: Paradigm dependent or independent? J Fluency Disord 1993; 18: 133-149.

[9] Norris J, Hoffman P. Whole language intervention for school age children. San Diego: Singular; 1993.

[10] Trautman L, Healey CH and Norris J. The effects of contextualization on fluency in three groups of children. J Lang Hear Res 2001; 44: 564-576.

[11] Hoffman P. Clinical forum: Phonological assessment and treatment. Synergistic development of phonetic skill. Lang Speech Hear Serv Sch 1992; 23: 254-260.

[12] Norris J \& Damico J. Whole language in theory and practice: Implication for Language Intervention. Lang speech hear serv sch 1990; 21: 212-220.

[13] Yairi E \& Ambrose N. Early childhood stuttering. Austin, Tx:ProEd; 1999.

[14] Hill D. Differential treatment of stuttering in early stages. In Gregory H. Ed, Stuttering therapy: rationale and procedures. Boston, M.A.: Allyn \& Bacon; 2003.

[15] Dinville C. La Tartamudez. Barcelona: Masson; 1997.

[16] Goodman K. What's whole in whole language?. Portsmouth, USA; NH. Heinemann; 1986. 